\title{
Conducted and segmental components of the somatosensory cervical response
}

\author{
M A S IM O LEANDRI, EMILIO F A V A LE, SANDRO RATTO, \\ A N D M CHELE A B B R UZESE
}

From the Department of Neurology, University of Genoa, Genoa, Italy

SUMMARY Cervical responses evoked by stimulation of the median nerve have been concurrently recorded from C7-Fz and C7-Sn (suprasternal notch). The existence of two different waveforms (RI and RII) has been confirmed. RI (from C7-Fz) consists of four negative peaks (N9, N11, N13, N14) followed by a large positive deflection (P16). RII (from C7-Sn) is characterised by an early positive-negative spike (P1-N1a) followed by a slow negative-positive wave (N1b-P2). The study of the most relevant parameters (polarity, latency and refractory period) of each component of RI and RII did not indicate whether the generators underlying RI differ from those responsible for RII. However, stimulation of the lower limb, which does not involve segmental events at cervical level, showed a clearcut difference: no response was recorded from C7-Sn, while evoked activity equivalent to RI was obtained from C7-Fz. Therefore it is suggested that RII is entirely generated by segmentally evoked potentials while RI is mainly due to conducted potentials.

Two waveforms of the somatosensory response recorded from the surface of the neck upon stimulation of the upper limb have been described. The first one (RI), investigated by a number of authors, ${ }^{1-4}$ consists of four negative peaks (N9, N11, N13 and N14) followed by a large positive deflection (P16). Such a response is usually obtained from the active electrode placed over the spinal column and a mid-frontal reference electrode $(\mathrm{Fz})$. Other reference electrode positions, more or less distant from the active one, have been used (for example hand, knee), without remarkable changes in latency or in polarity of the single components, with the only exception of N9. ${ }^{56}$ The second type of cervical response (RII), studied almost exclusively by a Japanese group, ${ }^{7}$ is characterised by an early positive-negative deflection followed by a negative-positive wave, the reference electrode being placed at the suprasternal notch $(\mathrm{Sn})$.

Read in part at the Meeting of the Società Italiana di Elettroencefalografia e Neurofisiologia held in $S$ Margherita Ligure on 14-15 November 1980.

Address for reprint requests: Massimo Leandri MD, Clinica Neurologica, Via De Toni, 5, I 16132 Genova, Italy.

Accepted 18 May 1981
It should be pointed out that no component of the RI response (loosely called the electrospinogram) is regarded as strictly spinal in origin, with the exception of N11, which is probably due to the conducted activity of the dorsal columns. ${ }^{2} \mathrm{~s}$ N9 is generally attributed to the activity of the brachial plexus, ${ }^{2}$ while N13 is thought to be generated by the dorsal column nuclei. ${ }^{2}{ }^{2} \mathrm{~N} 14$ and P16 are far field reflections of the activity of supraspinal somatosensory afferent pathways, from the medulla to the cerebral cortex, ${ }^{9}$ although a partial contamination from spinal segmental events cannot be excluded. The RI cervical response therefore mainly reflects the conduction of afferent impulses along the lemniscal pathway, from the brachial plexus to the thalamo-cortical projections.

In contrast, RII has been regarded as a reflection of spinal segmental events. ${ }^{7}$ In particular, the early positive-negative deflection may represent the incoming volleys conducted through the cervical roots, while the subsequent negativepositive wave is believed to reflect interneuronal activity and the related primary afferent depolarisation.

The possible relationships between these two responses have never been properly investigated. 
In particular, it is not clear at present whether the generators underlying RI do differ from those responsible for RII, or whether RI and RII are to be considered as different features of the same events, although the former explanation seems to be the most likely on the basis of the spatial orientation of the two derivations. To clarify this issue simultaneous recordings of RI and RII upon stimulation of the median nerve were performed, and the most relevant parameters of each component (that is polarity, latency and refractory period) of RI and RII were studied in a group of normal subjects. Moreover, stimulation of the lower limb, which does not exite the occurrence of segmental events at the cervical level, was performed and the relevance of the recording electrodes arrangement on the features of the evoked responses evaluated. Finally, additional information was obtained by leads suitably positioned over the shoulder, that is at different levels of the brachial plexus.

\section{Material and methods}

Observations were made on eight volunteer subjects, ranging in age from 26 to 39 years. Subjects were kept relaxed on a couch in a quiet room. The median and the tibial nerves were stimulated at the wrist and at the popliteal fossa respectively, using surface electrodes (Disa 13K62) with the cathode proximal to the anode. The limbs were gently warmed by an infrared lamp throughout the experiment to ensure a skin temperature of $36-38^{\circ} \mathrm{C}$. Square pulses were generated by a Disa 14E01 Ministim stimulator (pulse duration $0.2 \mathrm{~ms}$ ) at a rate of $1 \mathrm{~Hz}$. The stimulus intensity, both at the wrist and at the popliteal fossa,

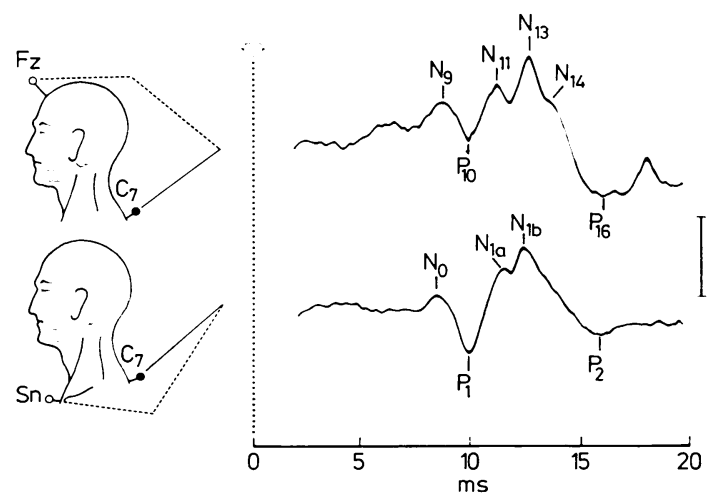

Fig 1 Cervical responses simultaneously recorded from $\mathrm{C7}-\mathrm{Fz}$ and $\mathrm{C7}-\mathrm{Sn}$ following median nerve stimulation at the wrist. In this and the following figures positivity is downwards. Calibration: $0.5 \mu \mathrm{V}$; 512 responses were summated. was three to four times the motor threshold of the innervated muscles. Both neck and shoulder recordings were performed by means of fine subcutaneous needles, with electrode impedance of 1000-2000 ohms. Five derivations were concurrently employed to record the activity evoked by stimulation of the upper limb, the electrodes being placed as in figs 1 and 2 . Cervical activity evoked by stimulation of the lower limb was simultaneously recorded by derivations C7$\mathrm{Fz}$ and $\mathrm{C7}-\mathrm{Sn}$ (fig 3). The input from the recording electrodes was led to differential amplifiers (Evoked potentials amplifiers Ote 2305) and simultaneously recorded on magnetic tape (Philips Analog 7): the output, summated by a digital averager (Neuroaverager Ote) triggered by the stimulator, was displayed on an X-Y plotter (Philips PM 8125). The frequency response of the recording apparatus was $16-10000 \mathrm{~Hz}(3 \mathrm{~dB})$. Analysis times of 20 or $50 \mathrm{~ms}$ were used and usually 512 or 1024 responses were summated. Number, shape and polarity of the recorded potentials were analysed visually. Peak latencies were measured on the plot or with a cursor on the computer oscilloscope.

\section{Results}

The general waveform of RI and RII (fig 1) was essentially similar to the original descriptions, apart from two additional peaks referred as P10

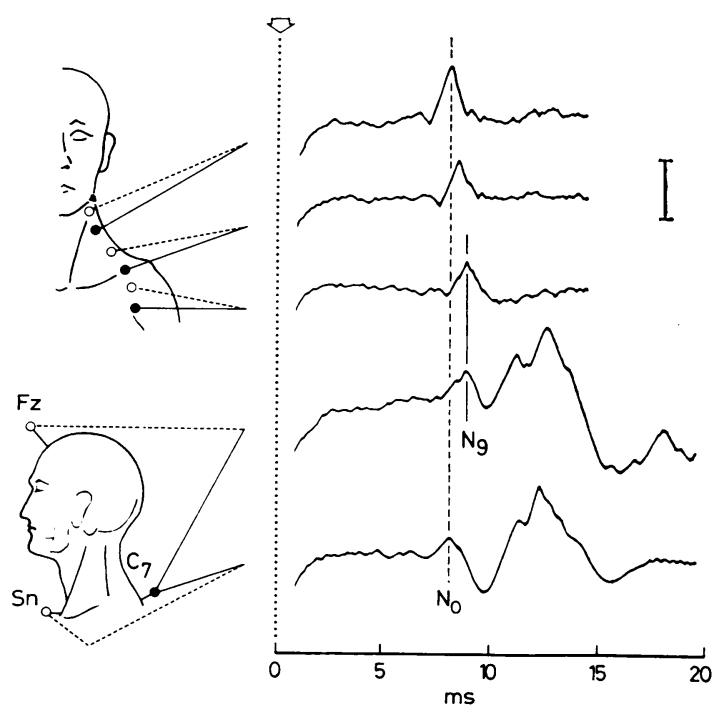

Fig 2 Neck and shoulder simultaneous recordings following median nerve stimulation at the wrist. The negative peak of the response recorded at the axilla (first trace) is coincident with NO, while the negative peak of the response recorded along the upper edge of the trapezius muscle (third trace) is coincident with N9. Calibration: $0.5 \mu \mathrm{V} ; 512$ responses were summated. 


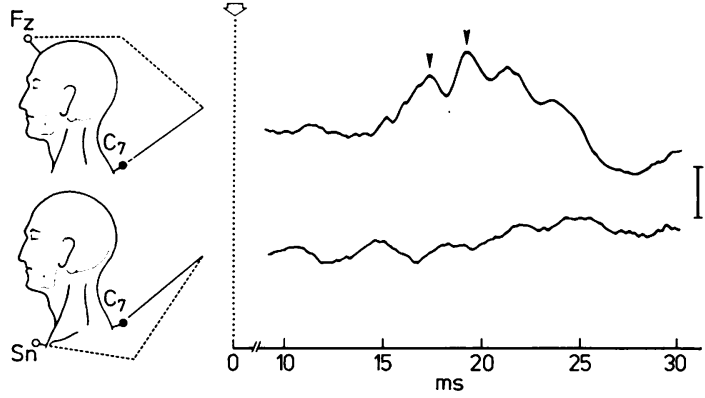

Fig 3 Neck recordings following tibial nerve stimulation at the popliteal fossa. C7-Fz picks up a clearcut response while C7-Sn fails to demonstrate any definite evoked activity. Arrows indicate the two negative peaks supposed to be equivalent to N11 and $N 13$ components of the cervical response evoked by stimulation of the upper limb. Calibration: $0.5 \mu \mathrm{V}$; 1024 responses were summated.

Table Mean latencies ( $m s$ ) of RI and RII components

\begin{tabular}{|c|c|c|c|c|c|c|}
\hline RI & & $\begin{array}{l}\text { N9 } \\
9 \cdot 3 \pm 0 \cdot 7\end{array}$ & $\begin{array}{l}\text { P10 } \\
10 \cdot 1 \pm 0 \cdot 8\end{array}$ & $\begin{array}{l}\text { N11 } \\
11 \cdot 4 \pm 1.0\end{array}$ & $\begin{array}{l}\mathrm{N} 13 \\
13 \cdot 1 \pm 0 \cdot 9\end{array}$ & $\begin{array}{l}\text { P16 } \\
16 \cdot 4 \pm 1 \cdot 0\end{array}$ \\
\hline RII & $\begin{array}{l}\Delta \\
\mathrm{p}\end{array}$ & $\begin{array}{l}\text { No } \\
8 \cdot 7 \pm 0.8 \\
0.6 \pm 0.1 \\
<0.001\end{array}$ & $\begin{array}{l}\text { P1 } \\
9.9 \pm 0.8 \\
0.1 \pm 0.2 \\
\text { ns }\end{array}$ & $\begin{array}{l}\text { Nla } \\
11 \cdot 5 \pm 0.8 \\
0.1 \pm 0.5 \\
\text { ns }\end{array}$ & $\begin{array}{l}\text { N1b } \\
12.7 \pm 0.9 \\
0.5 \pm 0.3 \\
<0.005\end{array}$ & $\begin{array}{l}\text { P2 } \\
16 \cdot 2 \pm 1 \cdot 0 \\
0 \cdot 2 \pm 0 \cdot 5 \\
\text { ns }\end{array}$ \\
\hline
\end{tabular}

N14 component has been omitted as RII does not show any peak at this time or occurring between N1b and P2.

and N0 respectively.* The mean latencies of the single components of RI and RII as well as their statistical parameters (paired $t$ test) are shown in the table. The brachial plexus response has been recorded at different levels of the shoulder. The negative peak of the triphasic (pnp) response was coincident with NO when picked up at the axilla, and with N9 when picked up along the upper edge of the trapezius muscle (fig 2).

Double pulse experiments showed that N9, P10, and N11 as well as N0, P1, and N1a, had a very short refractory period. When equal paired stimuli were given to the median nerve with different interstimulus intervals, a complete recovery could be observed within $2 \mathrm{~ms}$. Therefore it has been concluded that all these components are presynaptic in origin. As to the refractoriness of the subsequent ones it can be maintained that both N13

*The nomenclature originally proposed by Shimoji et $a^{10}$ for the cord dorsal potential (that is P1, N1, and P2) and subsequently extended? to the surface recorded response (that is RII) has been partially modified. Unlike these authors", we have called N1a and N1b the two distinct peaks following P1, N1b corresponding to the second component of the negative wave (cf Shimoji et al, ${ }^{7}$ the peak indicated by the arrow in the left side of fig 1 and the upper half of fig 2 ). and $\mathrm{N} 1 \mathrm{~b}$ are postsynaptic in nature, their complete recovery never requiring less than $5 \mathrm{~ms}$. No definite conclusions could be reached on the later components (that is P16 and P2), partly owing to their variability, but it seems very likely that they are to be considered postsynaptic in origin.

Cervical activity evoked by stimulation of the tibial nerve at the popliteal fossa was concurrently recorded from $\mathrm{C} 7-\mathrm{Fz}$ and $\mathrm{C7}-\mathrm{Sn}$. A clearcut response could be obtained from $\mathrm{C} 7-\mathrm{Fz}$ (fig 3), its general waveform being very similar to RI, as previously reported by Leandri et al. ${ }^{11}$ Moreover it was observed that the interwave latency between the first two negative peaks $(1 \cdot 6 \pm 0.3 \mathrm{~ms})$ was very close to the time interval between $\mathrm{N} 11$ and N13 $(1 \cdot 4 \pm 0.3 \mathrm{~ms})$.

In contrast no definite response (fig 3 ) could be detected from C7-Sn, even when more than 2000 replications were summated.

\section{Discussion}

Previous descriptions of the two waveforms of the somatosensory response recorded from the surface of the neck upon stimulation of the upper limb have been confirmed by our findings. This holds true also for the initial small negative deflection of RII, immediately preceding the P1-N1a diphasic complex, already recorded but apparently disregarded by Shimoji et al. ${ }^{7}$ Indeed such a deflection, tentatively called NO, is not visible in the cord dorsal potential (CDP) concurrently recorded from the posterior epidural space (cf Shimoji et al, ${ }^{7}$ left side of fig 1 and upper half of fig 2). It should be pointed out, however, that RII, in contrast to the CDP, has been obtained by a referential derivation which picks up both local and far field events. We presume that NO may originate from the most distal portion of the brachial plexus, since the concurrent recordings performed over the shoulder have shown that N0 has the same latency as a wave generated at the axilla, more peripherally than the origin site of N9.

A comparative analysis of RI versus RII, reveals that the P1 component occurs at the same time as P10. Usually such a peak is not evident as a distinct component of RI, possibly because of its small amplitude, but we feel that it should be identified with P1 (see also Abbruzzese et al ${ }^{8}$ ), although synchrony between two events cannot be regarded as a proof of a common origin.

It has been previously assumed ${ }^{2} 8$ that $\mathrm{N} 11$ and N13 components reflect the activity of dorsal columns and dorsal columns nuclei respectively: 
however simultaneous recordings of RI and RII recently performed by Dimitrijevic et al, ${ }^{12}$ challenge such a hypothesis. According to these authors, ${ }^{12} \mathrm{~N} 11$ and $\mathrm{N} 13$ are due respectively to presynaptic and postsynaptic activity in the dorsal horn neurons, N11 being equivalent to N1a and N13 to N1b.* Actually there is little doubt that $\mathrm{N} 11$ and N1a are presynaptic while N13 and N1b are postsynaptic in origin, as we demonstrated by double pulse experiments. However, while the mean latency of $\mathrm{N} 11$ is extremely close to that of N1a, N1b occurs significantly earlier than N13, more specifically in coincidence with the CDP N1 component. ${ }^{7}$

The difference in latency between N13 and N1b is in keeping with the assumption that $\mathrm{N} 13$ is generated at a more rostral level than N1b, possibly by the dorsal columns nuclei. The equivalence proposed by Sedgwick (personal communication) that $\mathrm{N} 11=\mathrm{N} 1 \mathrm{a}$ and $\mathrm{N} 13=\mathrm{N} 1 \mathrm{~b}$, and its functional implications, must be considered with caution although such a hypothesis cannot be definitely ruled out unless further evidences become available. The possible equivalence between P16 and P2 cannot be excluded: in fact both components are related to postsynaptic events and their mean latencies are not different.

In general, a constant gap in latency between two events can support the assumption that they are generated by different structures or by the same structure at different levels, while identical latencies are not by themselves a proof of the common origin of the two events, a mere coincidence between them being possible. This concept might hold true also for our findings: in fact we have not been able to demonstrate a clearcut difference between RI and RII either on the basis of their chronological features or by analysis of other relevant parameters. $\dagger$

Nevertheless, it is conceivable that one derivation (that is C7-Sn) could be selective with respect to generators at the cervical level, the spatial orientation of its electrodes being particularly suited to detect horizontal dipoles and to ignore vertical ones. Such assumption can be verified by stimulation of the lower limb, which is a suitable way to evoke conducted events in the absence of segmental cervical responses. In previous experiments ${ }^{11}$ we have shown some clearcut analogies between the response evoked by stimulation of the upper limb and that obtained by stimulation of the

*N1a and N1b correspond respectively to N3 and N4 components as described by Dimitrijevic et al.12

tActually it might be of relevance that on no occasion did RII recordings show a distinct peak chronologically related to N14 or occurring between N1b and P2. lower limb when the derivation $\mathrm{C} 7-\mathrm{Fz}$ is employed. In particular, apart from the expected absence of N9, we observed that the first negative peak of the response evoked by stimulation of the tibial nerve is due to a travelling wave arising in the most rostral portion of the dorsal columns, while the second negative peak is generated by a fixed dipole, probably located at level of the dorsal column nuclei. Since the interwave latency of these two peaks is very similar to the interval between $\mathrm{N} 11$ and N13 it seemed appropriate to assume that the first two negative peaks of the cervical response evoked by stimulation of the lower limb are equivalent to N11 and N13 respectively. ${ }^{11}$

The present investigation shows that the cervical activity evoked by stimulation of the lower limb can be recorded by C7-Fz but not by C7-Sn. We think that the former derivation is particularly suitable to pick up conducted events, probably generated by vertically oriented dipoles, though the co-detection of spinal segmental events cannot be excluded. On the other hand, the clearcut absence of any response upon stimulation of the lower limb seems to suggest that C7-Sn cannot pick up conducted events. It follows that the response recorded by C7-Sn upon stimulation of the upper limb (that is RII) is likely to be mainly (or exclusively) related to segmental events generated by dipoles which are horizontally oriented, as suggested by the maximal amplitude of the CDP in the anteroposterior axis on the horizontal plane. ${ }^{13}$

We conclude that the electrophysiological events underlying RI cannot be identified with those responsible for RII. In this connection it seems of relevance that in spinal man RII is still present, ${ }^{14}$ while only the initial portion of RI (that is N9 and N11) can be recorded after a complete spinal cord lesion ${ }^{3}$ at C5. Further investigations in patients with high cervical lesions are still required, however, since good recordings from such patients are technically difficult to obtain. ${ }^{15}$

\section{References}

1 Matthews WB, Beauchamp M, Small DB. Cervical somatosensory evoked responses in man. Nature 1974; 252:230-2.

2 Jones SJ. Short latency potentials recorded from the neck and scalp following median nerve stimulation in man. Electroencephalogr. Clin Neurophysiol 1977; 43:853-63.

3 El-Negamy E, Sedgwick EM. Properties of a spinal somatosensory evoked potential in man. 
J Neurol Neurosurg Psychiatry 1978; 41:762-8.

4 Abbruzzese $M$, Favale E, Leandri M, Ratto S. Spinal components of the cerebral somatosensory evoked response in man: the "S wave". Acta Neurol Scand 1978; 58:213-20.

5 Cracco RQ, Cracco JB. Somatosensory evoked potentials in man: far field potentials. Electroencephalogr Clin Neurophysiol 1976; 41:460-6.

6 Yamada T, Kimura J, Nitz DN. Short latency somatosensory evoked potentials following median nerve stimulation in man. Electroencephalogr Clin Neurophysiol 1980; 48:367-76.

7 Shimoji K, Shimizu H, Maruyama Y. Origin of somatosensory evoked responses recorded from the cervical skin surface. J Neurosurg 1978; 48: $980-4$.

8 Abbruzzese $M$, Favale $E$, Ivaldi $M$, Leandri $M$, Ratto S. Nuove acquisizioni sulla risposta cervicale somestesica. Riv Ital Elettroencef Neurofisiol 1979; 2:599-603.

9 Abbruzzese M, Favale E, Leandri M, Ratto S. Ricerche sulle componenti encefaliche del potenziale spinale evocato da stimolazione del nervo mediano nell'umo. Boll Soc Ital Biol Sper
1978; 54:1925-30.

10 Shimoji K, Kano T, Higashi $\mathrm{H}$, Morioka T, Henschel EO. Evoked spinal electrograms recorded from epidural space in man. J Appl Physiol 1972; 33:468-71.

11 Leandri M, Ratto S, Abbruzzese M, Favale E. Ricerche sul potenziale cervicale evocato da stimolazione del nervo tibiale. Riv Ital Elettroencef Neurofisiol 1980; (in press).

12 Dimitrijevic MR, Sedgwick EM, Sherwood A. A spinal cord potential in man. J Physiol (London) 1980; 303:37P.

13 Shimoji K, Matzuki M, Shimizu H. Wave-form characteristics and spatial distribution of evoked spinal electrogram in man. $J$ Neurosurg 1977; 46: 304-13.

14 Shimoji K, Kano T, Morioka T, Irezon E. Evoked spinal electrogram in a quadriplegic patient. Electroencephalogr Clin Neurophysiol 1973; 35: 659-62.

16 Sedgwick EM, El-Negamy E, Frankel H. Spinal cord potentials in traumatic paraplegia and quadriplegia. J Neurol Neurosurg Psychiatry 1980; 43:823-30. 